\title{
Lysyloxidase-4 is a selectively expressed candidate diagnostic antigen in pancreatic cancer
}

\author{
Marian Kiel ${ }^{1}$, Elgar Susanne Quabius ${ }^{1,2}$, Christian Röder ${ }^{3}$, Petra Ambrosch $^{1}$, Markus Hoffmann ${ }^{1}$ and Tibor Görögh ${ }^{1 *}$ \\ ${ }^{1}$ Department of Otorhinolaryngology, Head and Neck Surgery, University of Kiel, Kiel, Germany \\ ${ }^{2}$ Institute of Immunology, University of Kiel, Kiel, Germany \\ ${ }^{3}$ Division of Molecular Oncology, Institute for Experimental Cancer Research, University of Kiel, Kiel, Germany
}

\begin{abstract}
Lysyl oxidases (LOX) catalyze collagen and elastin cross-linking but also facilitate tumor genesis and progression. In this study, we used a monoclonal antibody directed against LOX like-4 (LOXL-4) on pancreatic cancer tissue specimens of 103 patients to examine its diagnostic and prognostic value. In $88.3 \%$ of pancreatic cancer specimens, LOXL-4 was detectable, whereas it could not be shown in benign oral mucosa control specimens. The level of LOXL-4 expression strongly correlated with $\mathrm{T}$ stage and lymph node status but not with occurrence of distant metastases or overall patient survival. These results substantially strengthen the existing immunohistochemical studies on LOXL-4 in different malignomas and clearly suggest a diagnostic value of LOXL-4 in pancreatic carcinoma and do contribute to the ongoing discussion about to what extent LOXL-4 might be a suitable marker in cancer diagnosis and progression.
\end{abstract}

Abbreviations: LOXL: Lysyl Oxidase-Like; TNM: Tumor, Nodes, Metastases; FCS: Fetal Calf Serum

\section{Introduction}

Due to the aggressive nature and disadvantageous prognosis, patients suffering from pancreatic cancer still show 5-year survival of as little as $1 \%$ [1] and survival time of a few months only [2]. Both incidence and mortality have barely changed over the past 20 years, with diagnosis still happening late [3]. Consequently, it is desirable to improve diagnosis and enable an earlier start of therapy, possibly by determine new markers for (early) diagnosis.

Lysyl oxidases are a group of enzymes best known for catalyzing cross-linking of collagen and elastin [4]. They mediate important activities in tumor genesis and progression, such as increased cellular growth and cell migration [5]. In gastric cancer it was shown that LOX-family enzymes catalyze cellular invasion in surrounding tissue [6]. The study presented here aimed to examine the LOXL-4 expression in pancreatic cancer and analyze its possible prognostic and diagnostic value by means of immunohistochemistry. For this purpose, we examined 103 confirmed pancreatic cancer specimens immunohistochemically. LOXL-4 expression was correlated with tumor stage, lymph node status, occurrence of distant metastasis and overall patient survival.

\section{Material and methods}

\section{Patients}

Patient characteristics were as follows: 57 (55.3\%) males; 46 (44.7\%) females. Age at diagnosis: 35.1 to 85.3 years, median age: 65.4 years. After surgery patients were followed until death or until September 2013, median follow-up: 6.31 years (range 0.12 to 14.24 years). Tissue samples were obtained between 1999 and 2013 during surgery at the University Medical Center Schleswig Holstein (UKSH) Campus Kiel. Fresh frozen samples of histopathologically confirmed pancreatic cancer $(n=103)$ were available for this study. All samples were obtained following informed consent; the study was approved by the local Ethics Committee (A 110/99). The TNM classification and staging was performed according to the UICC criteria from 2002. The data necessary for statistical evaluation were obtained from the UKSH Campus Kiel data base.

\section{Immunohistochemistry}

The fresh frozen sections $(4-5 \mu \mathrm{m})$ were dried; the endogenous peroxidase was blocked by $100 \mathrm{ml}$ methanol plus $1 \mathrm{ml} 30 \%$ hydrogen peroxide. Remaining enzyme activity was inhibited by applying a fetal calf serum (FCS) / phosphate buffer saline (PBS) solution (60 $\mu$ FCS in $2 \mathrm{ml}$ PBS [0.15 NaCl, 2.5mM KCl, 15mM NaH2PO4, 15mM Na2HPO4, pH 7.4]).

Primary monoclonal anti-LOXL-4 antibody (in-house production) was added to the samples for $1 \mathrm{~h}$ at room temperature, followed by incubation with a biotin-conjugated secondary antibody (Dako, Hamburg, Germany) at room temperature for $1 \mathrm{~h}$. The optimum concentration of the primary antibody $(1: 400)$ was determined using serial dilutions of highly purified synthetic peptide as described previously $[7,8]$.

A labeled peroxidase complex system (ABC-Vectorstatin, Dako, Hamburg, Germany) was used to visualize all immune reactions. Sections were counterstained with Mayer's haematoxylin. Negative controls were obtained by omission of the primary antibody and by incubating the primary antibody with specific blocking peptide in a five- to ten-fold molar excess before staining.

Correspondence to: Tibor Görögh, Department of Otorhinolaryngology, Head and Neck Surgery, University of Kiel, Kiel, Germany, Tel: +49 43150021 850, E-mail: Tibor.Goeroegh@uksh.de,

Key words: pancreatic carcinoma, LOXL-4, immunohistochemistry

Received: May 02, 2017; Accepted: May 18, 2017; Published: May 22, 2017 
The evaluation of the specimens was performed by two independent examiners who were not familiar with the clinical records using a light microscope (Zeiss Axioplan, Hamburg). For assessing of the histological specimens, the portion of positively stained cells in five areas was determined at $\times 400$ magnification, a total of 300 cells per specimen were analyzed. Afterwards the specimens were grouped in four different categories according to the percentage of positively stained cells: Negative $<5 \%$, weak $5-30 \%$, moderate $31-75 \%$ and strong $>75 \%$. For statistical analysis negative and weak and moderate and strong samples were pooled respectively.

\section{Statistical analysis}

Immunohistochemical data were analyzed using Fisher's exact test followed by chi-square test (SPSS 20.0). For Kaplan-Meier survival analysis the primary statistical end point was overall survival; defined as time from diagnosis to date of last follow-up or death. Testing the prognostic value of LOXL-4 expression Log rank tests were performed and $p$-values $\leq 0.05$ defined significant differences between groups.

\section{Results}

\section{Distribution of LOXL-4 expression in pancreatic carcinomas}

LOXL-4 expression could be detected in 91 (88.3\%) of the 103 examined pancreatic cancer specimens. Of these 91 positive cases 24 (23.3\%) showed weak, 32 (31.7\%) moderate and 35 (34.0\%) strong LOXL-4 expression respectively. Typical staining patterns of negative, weak, moderate and strong LOXL-4 expression is depicted in Figure1.

\section{LOXL-4 expression correlates with $\mathrm{T}$ stage}

The distribution of the LOXL-4 expression pattern in relation to T-status of the tumors is shown in Figure 2. Due to incomplete data comparison of LOXL-4 expression and T stage could only be performed for 95 samples. For statistical analysis, tumors with negative and weak expression and tumors with moderate and strong expression were grouped. Likewise, tumors with $\mathrm{T} 1$ and $\mathrm{T} 2$ stage were pooled and tumors with $\mathrm{T} 3$ and $\mathrm{T} 4$ stage were pooled. Of the 95 pancreatic cancer specimens 8 (8.4\%) samples were T1/T2 with negative / weak LOXL-4 expression, 23 (24.1\%) samples were T3/T4 showing negative / weak LOXL-4 expression. Further 4 (4.2\%) samples were T1/T2 with moderate / strong LOXL-4 expression and the remaining 60 (63.2\%) samples were T3/T4 with moderate / strong LOXL-4 expression $(\mathrm{p}=0.011)$.

\section{LOXL-4 expression correlates with lymph node status}

The distribution of the LOXL-4 expression pattern in relation to lymph node status of the tumors is shown in Figure 3. Comparison of LOXL-4 expression and lymph node status could only be performed for 96 samples due to incomplete data. For statistical analysis, tumors with negative and weak expression and tumors with moderate and strong expression were again pooled. Of the 96 pancreatic cancer specimens $16(16.7 \%$ samples each were either $\mathrm{N}[0]$ or $\mathrm{N}[1]$ and showed negative / weak LOXL-4 expression. Among the samples with moderate / strong LOXL-4 expression 10 (10.4\%) were N[0] and 54 (56.3\%) N[1] $(\mathrm{p}=0.001)$.

\section{LOXL-4 expression does not correlate with the occurrence of distant metastases}

Comparison of LOXL-4 expression and occurrence of distant metastases was performed for 98 samples due to incomplete data. For statistical analysis, tumors with negative and weak expression
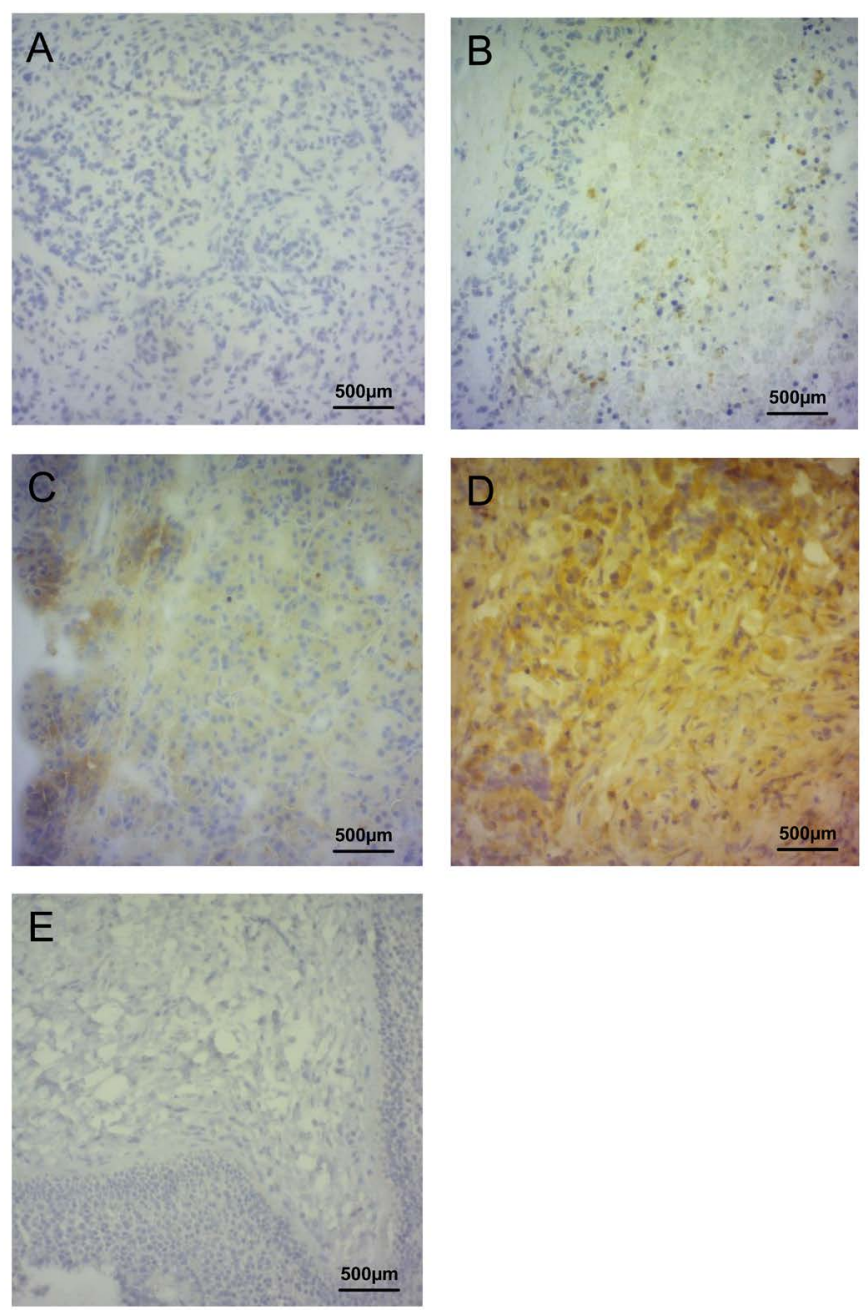

Figure 1. Immunohistochemical staining of LOXL-4.

Figures 1A to 1D show, negative (A), weak (B), moderate (C) and strong immunohistochemical staining of LOXL-4 in pancreatic cancer specimens. Figure 1 E shows benign oral mucosa specimen serving as negative control. Magnification of all images was 200 .

and tumors with moderate and strong expression were grouped. Of the 98 cancer specimens $28(28.6 \%)$ were $\mathrm{M}[0]$ with negative / weak LOXL-4 expression and 4 (4.1\%) were M[1] showing negative / weak LOXL-4 expression. Of those samples with moderate / strong LOXL-4 expression 58 (59.2\%) were M[0] and 8 (8.2\%) were $\mathrm{M}[1]$ ( $\mathrm{p}=0.597)$.

\section{LOXL-4 expression does not correlate with overall patient survival}

To not only study the possible diagnostic value of LOXL-4, we next correlated LOXL-4 expression with overall patient survival to determine its potential prognostic value. However, again due to incomplete data files, survival data could only be calculated for 76 patients. As shown in Figure 4 no significant correlation between LOXL-4 expression and overall patient survival was found $(\mathrm{p}=0.715)$ when grouping negative and weak stained tumors versus moderate and strong stained ones. Two-year survival rates for patients negative and weak as wells as moderate and strong LOXL-4 expression was both 36.5\%. The 5-year survival rates for patients with negative and weak LOXL-4 expression was $0 \%$, whereas it was $6.1 \%$ for patients with moderate and strong LOXL-4 expression. 


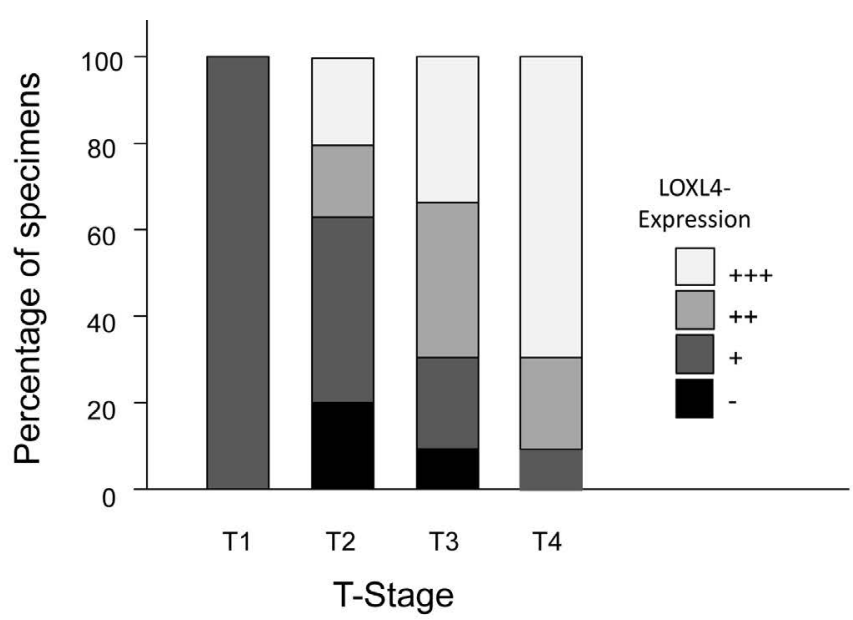

Figure 2. LOXL-4 expression in correlation with T stage.

Immunohistochemical staining of LOXL-4 in pancreatic cancer specimens in relation to $\mathrm{T}$ stage are shown. Black parts of the columns indicate negative, dark grey areas weak, grey ones moderate and light grey areas strong LOXL-4 staining.

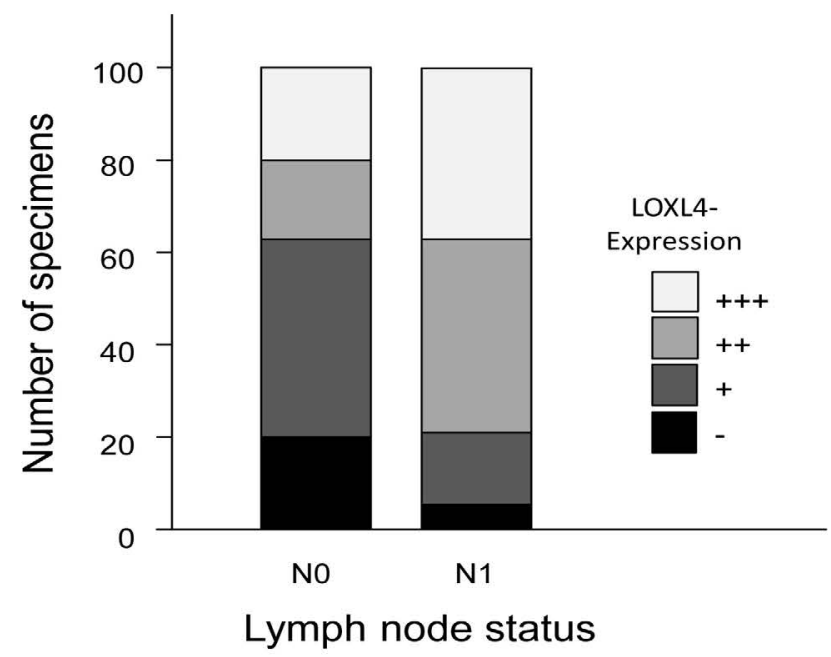

Figure 3. LOXL-4 expression in correlation with lymph node status.

Immunohistochemical staining of LOXL-4 in pancreatic cancer specimens in relation to lymph node status are shown. Black parts of the columns indicate negative, dark grey areas weak, grey ones moderate and light grey areas strong LOXL-4 staining.

\section{Discussion}

Previously we could show that LOXL-4 mRNA was highly upregulated in squamous head and neck cancers (HNSCC) when compared to normal epithelial cells [7], these results could be confirmed by immunohistochemistry. In addition a significant correlation between LOXL-4 expression and lymph node metastases and higher tumor stage was detected in HNSCC [8]. However, in patients with primary head and neck cancer LOXL-4 expression had no effect on overall survival although patients with highly positive LOXL-4 nodal metastases showed prolonged albeit not significant overall survival [7].

In the study presented we examined LOXL-4 expression, by means of immunohistochemistry, in 103 patients with pancreatic cancer and correlated the expression data with TMN stages of the tumors and performed Kaplan-Meyer analysis to correlate the expression data with overall survival of the patients. Overall LOXL-4 was present in $88.3 \%$ of pancreatic cancer specimens, with similar results being found in HNSCC [8]. Similar, as previously shown for HNSCC [8] tumor staging was significantly correlated with increased LOXL-4 expression in the here analyzed pancreatic cancer specimens. Different results, however, were obtained in a breast cancer mouse model, where LOXL4 knockdown resulted in enhanced tumor growth [ $\left.{ }^{9}\right]$. Correlating LOXL-4 expression with lymph node status also revealed a positive correlation between these two factors, again with similar results seen in HNSCC [8] and in a rat model for prostate cancer [10]. Interestingly, no correlation between LOXL-4 expression and the occurrence of distant metastases could be found (Tables 1-5). This is in contrast to our previous data obtained in HNSCC [8] but might contribute to the ongoing discussion regarding the either tumor promoting or tumor suppressing role of LOXL-4 in different cancer entities. For example HNSCC showed increased LOXL-4 expression after becoming invasive and metastasizing, when compared to non-invasive and non-

Table 1. Distribution of LOXL4 expressions in pancreatic carcinomas.

\begin{tabular}{|c|c|c|}
\hline \multicolumn{2}{|c|}{ Expression level } & LOXL4 \\
\hline \multirow{2}{*}{ Low $<30 \%$} & - & $12(11.7 \%)$ \\
\cline { 2 - 3 } & + & $24(23.3 \%)$ \\
\hline \multirow{2}{*}{ High $\geq 30 \%$} & ++ & $32(31.7 \%)$ \\
\hline Total & +++ & $35(34 \%)$ \\
\hline
\end{tabular}

Table 2. LOXL4 as a diagnostic marker for differentiation between low-grade and highgrade tumors.

\begin{tabular}{|c|c|c|c|c|c|c|}
\hline \multirow[b]{2}{*}{ LOXL4 } & \multicolumn{2}{|c|}{ T stage } & \multirow[b]{2}{*}{ Total [n] } & \multirow{2}{*}{$\begin{array}{c}\text { Sensitivity } \\
\text { (positive } \\
\text { predictive } \\
\text { value) }\end{array}$} & \multirow{2}{*}{$\begin{array}{c}\text { Specificity } \\
\text { (negative } \\
\text { predictive } \\
\text { value) }\end{array}$} & \multirow[b]{2}{*}{ P-Wert } \\
\hline & \begin{tabular}{|c|}
$\mathrm{T} 1 / \mathrm{T} 2$ \\
Low-grade \\
{$[\mathrm{n}]$}
\end{tabular} & $\begin{array}{c}\text { T3/T4 } \\
\text { High-grade } \\
\text { [n] } \\
\end{array}$ & & & & \\
\hline$-/+$ & $8(66.7 \%)$ & $23(27.7 \%)$ & $31(32.6 \%)$ & \multirow{2}{*}{$\begin{array}{c}72.3 \% \\
(81.8 \%)\end{array}$} & \multirow{2}{*}{$\begin{array}{c}66.7 \% \\
(53.8 \%)\end{array}$} & \multirow{2}{*}{0.011} \\
\hline$++/+++$ & $4(33.3 \%)$ & $60(72.3 \%)$ & $64(67.4 \%)$ & & & \\
\hline Total $[\mathrm{n}]$ & $12(100 \%)$ & $83(100 \%)$ & $95(100 \%)$ & & & \\
\hline
\end{tabular}

Table 3. LOXL4 as diagnostic marker for differentiation between positive and negative lymph node status.

\begin{tabular}{|c|c|c|c|c|c|c|}
\hline \multirow[b]{2}{*}{ LOXL4 } & \multicolumn{2}{|c|}{ Lymph node status } & \multirow[b]{2}{*}{ Total $[\mathbf{n}]$} & \multirow{2}{*}{$\begin{array}{c}\text { Sensitivity } \\
\text { (positive } \\
\text { predictive } \\
\text { value) }\end{array}$} & \multirow{2}{*}{$\begin{array}{c}\text { Specifity } \\
\text { (negative } \\
\text { predictive } \\
\text { value) }\end{array}$} & \multirow[b]{2}{*}{ P-Wert } \\
\hline & No [n] & $\mathrm{N} 1[\mathrm{n}]$ & & & & \\
\hline$-/+$ & $16(61.5 \%)$ & $16(22.9 \%)$ & $32(33.3 \%)$ & \multirow{2}{*}{$\begin{array}{c}77.1 \% \\
(84.3 \%)\end{array}$} & \multirow{2}{*}{$\begin{array}{l}61.5 \% \\
(50 \%)\end{array}$} & \multirow{2}{*}{0.001} \\
\hline$++/+++$ & $10(38.5 \%)$ & $54(77.1 \%)$ & $64(66.7 \%)$ & & & \\
\hline Total $[\mathrm{n}]$ & $26(100 \%)$ & $70(100 \%)$ & $96(100 \%)$ & & & \\
\hline
\end{tabular}

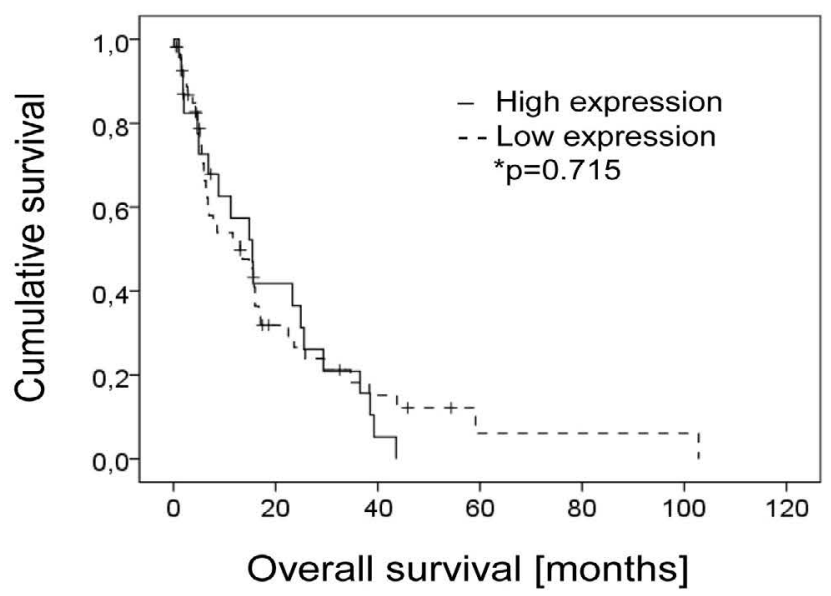

Figure 4. LOXL-4 expression in correlation with the occurrence of distant metastasis. Immunohistochemical staining of LOXL-4 in pancreatic cancer specimens in relation to the occurrence of distant metastasis are shown. Black parts of the columns indicate negative, dark grey areas weak, grey ones moderate and light grey areas strong LOXL-4 staining. 
Table 4. LOXL4 expression in relation to the occurrence of distant metastases.

\begin{tabular}{|c|c|c|c|c|c|c|}
\hline \multirow{2}{*}{ LOXL4 } & \multicolumn{2}{|c|}{ Presence of distant metastases } & \multirow{2}{*}{ Total [n] } & \multirow{2}{*}{$\begin{array}{l}\text { Sensitivity (positive } \\
\text { predictive value) }\end{array}$} & \multirow{2}{*}{$\begin{array}{l}\text { Specifity (negative } \\
\text { predictive value) }\end{array}$} & \multirow{2}{*}{ P-Wert } \\
\hline & M0 [n] & M1 [n] & & & & \\
\hline$-/+$ & $28(32.6 \%)$ & $4(33.3 \%)$ & $32(32.7 \%)$ & $66.7 \%$ & $32.6 \%$ & \multirow{2}{*}{0.597} \\
\hline$++/+++$ & $58(67.4 \%)$ & $8(66.7 \%)$ & $66(67.3 \%)$ & $(12.1 \%)$ & $(87.5 \%)$ & \\
\hline Total $[\mathrm{n}]$ & 86 & 12 & 98 & & & \\
\hline
\end{tabular}

Table 5. Overall patient survival in relation to LOXL4 expression levels.

\begin{tabular}{|c|c|c|c|c|c|c|}
\hline LOXL4 expression & Total number [n] & Events [n] & Censoring [n] & 2-year survival rate & $\begin{array}{c}\text { 5-year survival rate } \\
\text { Log-Rang test }\end{array}$ & $\begin{array}{c}\text { Mean survival time } \\
{[\text { months] }}\end{array}$ \\
\hline Low $<30 \%$ & 23 & $20(87 \%)$ & $3(13 \%)$ & $36.5 \%$ & $0 \%$ \\
\hline High $\geq 30 \%$ & 55 & $42(76,4 \%)$ & $13(23.6 \%)$ & $36.5 \%$ & 15.62 \\
\hline Total $[\mathrm{n}]$ & 78 & $62(79.5 \%)$ & $16(20.5 \%)$ & & 14.83 \\
\hline
\end{tabular}

metastasizing tumors [8]. In other studies, however, LOXL-4 showed tumor suppressive properties. Analyzing LOXL-4 expression in either normal human prostate specimens or in prostate malignancies revealed a loss of LOXL-4 expression during progression to metastasis [11]. Similarly in an animal model LOXL-4-knockout rat kidney fibroblasts showed tumor-like transformation exhibited carcinogenic effects when transplanted into mice [12]. Csiszar and coworkers showed that loss or reduction of LOX function during colon cancer development is a direct consequence of somatic mutations of the LOX gene within the $5 \mathrm{q} 23.1$ region and is associated with colon tumor pathogenesis, with loss or reduction of LOX function contributing to tumor progression [13].

The effect of LOXL-4 as a prognostic marker is also discussed with some controversy. In the study presented here, no significant correlation between LOXL-4 expression and overall survival of patients with pancreatic cancer could be seen, however $6.1 \%$ of the patients with moderate or strong LOXL-4 expression were still alive after 5 years whereas none of the patients with negative or weak LOXL4 expression were alive 5 years after diagnosis. Similarly, HNSCC patients with highly positive LOXL- 4 nodal metastases also showed prolonged albeit not significant overall survival [7]. In line with these observations are the results by Choi and coworkers, demonstrating a significant correlation between weak LOXL-4 expression and poor overall survival in breast cancer patients [9]. The same correlation between low LOXL-4 expression and overall survival rates and higher cumulative recurrence was reported in patients with hepatocellular carcinoma [14]. In nasopharyngeal cancer, on the other hand, high LOX expression correlated with decreases in 5-year survival, overall survival, distant metastasis-free survival and disease-free survival [ $\left.{ }^{15}\right]$. The reasons for the different patients' outcome dependent on LOXL-4 expression still remain unclear.

\section{Conclusion}

The data presented here investigating the role of LOXL-4 as prognostic and diagnostic marker in 103 histological confirmed pancreatic cancer specimens contribute to the ongoing discussion about to what extent LOXL-4 might be a suitable marker in cancer diagnosis and progression. The data strengthen the notion that the function of the LOX4 is context-dependent due to its either tumor-suppressing or tumor-promoting properties in different cancer entities. Therefore, further investigations are needed to understand the circumstances under which LOX4 may be set as a therapeutic target in cancer therapy.

\section{Authorship and contributorship}

This study was designed, directed and coordinated by M.K. and T.G. T.G., as the principal investigator, provided conceptual and technical guidance for all aspects of the project. E.S.Q., C.R. and M.H. performed the experiments and analyzed the data. P.A. and C.R. contributed reagents/materials/analysis tools. The manuscript was written by E.S.Q. and M.K. and commented on by all authors.

\section{Acknowledgements}

The authors thank Gudrun Scherer and Liane Carstensen for the preparation of the test specimens and the skillful technical assistance with the immunohistochemistry.

\section{Competing interests}

The authors declare that they have no competing interests.

\section{References}

1. Gudjonsson B (2009) Pancreatic cancer:survival, errors and evidence. Eur $J$ Gastroenterol Hepatol 21: S1379-S1382. [Crossref]

2. Li D1, Xie K, Wolff R, Abbruzzese JL (2004) Pancreatic cancer. Lancet 363: 1049 1057.[crossref]

3. Verma M (2005) Pancreatic cancer epidemiology. Technol Cancer Res Treat 4: 295 301.[crossref]

4. Kagan HM, Trackman PC (1991) Properties and function of lysyl oxidase. Am J Respir Cell Mol Biol5: 206-210.[crossref]

5. Csiszar K (2001) Lysyl oxidases: a novel multifunctional amine oxidase family. Prog Nucleic Acid Res Mol Biol 70: 1-32. [crossref]

6. Li RK, Zhao WY, Fang F, Zhuang C, Zhang XX, et al. (2015) Lysyl oxidase-like 4 (LOXL4) promotes proliferation and metastasis of gastric cancer via $\mathrm{FAK} / \mathrm{Src}$ pathway. J Cancer Res Clin Oncol 141: 269-281.[crossref]

7. Weise JB, Rudolph P, Heiser A, Kruse M, Hedderich J, et al. (2008) LOXL4 is a selectively expressed candidate diagnostic antigen in head and neck cancer. Eur $J$ Cancer 44: 1323-1331. [crossref]

8. Gorogh T, Weise JB, Holtmeier C, Rudolph P, Hedderich J, et al. (2007) Selective upregulation and amplification of the lysyl oxidase like-4 (LOXL4) gene in head and neck squamous cell carcinoma. $J$ Pathol 212: 74-82. [crossref]

9. Choi SK, Kim HS,JinT, Moon WK (2017) LOXL4 knockdown enhances tumor growth and lung metastasis through collagen-dependent extracellular matrix changes in triplenegative breast cancer. Oncotarget 8: 11977-11989. [crossref]

10. Nilsson M,Adamo H, Bergh A,Halin Bergstrom S (2016) Inhibition of lysyl oxidase and lysyl oxidase-like enzymes has tumour-promoting and tumour-suppressing roles in experimental prostate cancer. Scientific reports 6: 19608.

11. Ren C, Yang G, Timme TL, Wheeler TM, Thompson TC (1998) Reduced lysyl oxidase messenger RNA levels in experimental and human prostate cancer. Cancer Res 58: 1285-1290.[crossref]

12. Giampuzzi M, Botti G, Cilli M, Gusmano R, Borel A, et al. (2001) Down-regulation of lysyl oxidase-induced tumorigenic transformation in NRK-49F cells characterized by constitutive activation of ras proto-oncogene. J Biol Chem 276: 29226-29232.[crossref]

13. Csiszar K, Fong SFT, Ujfalusi A, Krawetz SA, Salvati EP, et al. (2002) Somatic mutations of the lysyl oxidase gene on chromosome $5 \mathrm{q} 23.1$ in colorectal tumors. Int J Cancer 97: 636-642. [crossref] 
14. Tian M, Liu W, Jin L, Jiang X, Yang L, et al. (2015) LOXL4 is downregulated in hepatocellular carcinoma with a favorable prognosis. Int J Clin Exp Pathol 8: $3892-$ 3900. [crossref]
15. HuaY, Wang H, Tang L, Chen Q, Shao J, et al. (2016) LOX expression in primary nasopharyngeal carcinoma. Correlation with prognostic parameters and outcome. Oncotarget 7: 8200-8207. [crossref]

Copyright: (C2017 Kiel M. This is an open-access article distributed under the terms of the Creative Commons Attribution License, which permits unrestricted use, distribution, and reproduction in any medium, provided the original author and source are credited. 